\title{
Pembrolizumab Treatment-Induced Liver Toxicity
}

\author{
Benoît Calderon Alma Stancu François-Roger Vanel Léa Vazquez \\ Institut Sainte Catherine, Avignon, France
}

\section{Keywords}

Anti-programmed cell death protein $1 \cdot$ Pembrolizumab $\cdot$ Immune-mediated hepatitis · Lung adenocarcinoma

\section{Abstract}

T cells play a critical role in immune responses against neoplasm. This finding contributed to the immunotherapy development, an effective treatment for many cancers nowadays. Programmed cell death protein 1 (PD1) is an inhibitory receptor on T cells which downregulate T-cell function per ligation with its ligands (PDL1 and PDL2). PD1 blockade is used to enhance antitumor immunity. Pembrolizumab is a humanized monoclonal anti-PD1 antibody currently used in the management of melanoma, non-small-cell lung cancer, and Hodgkin lymphoma. Most of the treatment toxicities are immune-related adverse events, but grade 3-4 toxicities occur in up to $5 \%$ of patients, mainly dermatologic. We present a case of grade 4 pembrolizumab-induced liver toxicity associated with an excellent treatment response in a Caucasian woman.

\section{Introduction}

Liver toxicity is a rare side effect associated with blockade of programmed cell death protein 1 (PD1), with $<1 \%$ of immune-related hepatitis observed in clinical trials of pembrolizumab [1]. Pembrolizumab is a human PD1-blocking antibody that has been extensively investigated in many cancers. It was approved by the US FDA for the treatment of advanced melanoma and non-small cell lung cancer (NSCLC) in 2015 and for the first-line treatment of patients with metastatic or unresectable recurrent head and neck squamous cell carcinoma (SCC) in 2019 [2].

This inhibitory receptor, by binding to its ligands, downregulates T-cell function by suppressing signaling pathways downstream of T-cell receptor (TCR) stimulation [3, 4]. PDL1 is expressed on the neoplastic cells of numerous cancers. By binding to PD1 on T cells leading 
to its inhibition, PDL1 expression is a major mechanism by which tumor cells can evade immune attack [1]. PDL1 overexpression may be intrinsic (related to cellular/genetic aberrations in these neoplastic cells) and/or adaptive (related to induction of PDL1 expression in the tumor environment, for example, in response to interferon $\gamma[5,6]$.

The use of checkpoint inhibitors has revealed a unique set of inflammatory toxicities termed irAEs. Pembrolizumab has been tested clinically in a series of KEYNOTE studies. Most of the related toxicities are irAEs, and grade 3-4 toxicities only occur in up to 5\% of patients [1]. Although these toxicities can appear in any organ, the most common are cutaneous, pulmonary, and colic. Hepatitis is generally related in $<1 \%$ of cases [1]. To date, cases of rare life-threatening or fatal irAEs have been reported after pembrolizumab therapy [7-13], but few reports immune-mediated hepatitis (IMH) toxicity, an uncommon complication of immune checkpoint inhibitor therapy, mostly mild and self-limited or in patients treated with a combination of immunotherapy [14]. We present a case of pembrolizumab-induced grade 4 acute immune-related hepatitis, a life-threatening irAE, in a Caucasian woman.

\section{Case Report}

A 56-year-old Caucasian woman, with a high tobacco (45 pack-year unweaned) and alcohol consumption ( 3 drinks/day $=90 \mathrm{~g} /$ day), suffered from stage 4 lung adenocarcinoma with pleural and adrenal metastases associated with T3N0M0 SCC of the tonsil. First was diagnosed clinically the tonsil SCC because of a progressive dysphagia, evolving for 2 months. A positron emission tomography-computed tomography was realized to evaluate the staging of this disease, which revealed an FDG-avid subscapular lymph node and 2 FDG-avid nodular thickening of the parietal pleura and the right adrenal gland, which were suspicious for distant metastases. Lymph node biopsy revealed the presence of tumor cells corresponding to an adenocarcinoma TTF1+ without EGFR mutation. Immunohistochemistry staining on SCC of the tonsil for $\mathrm{p} 16 / \mathrm{HPV}$ was positive, and immunohistochemistry staining on lung for PDL1 was highly positive (80\%) (Fig. 1). Clinically, the general condition is maintained with an ECOG Performance Status of 1. Physical examination allows visualizing the budding $4-\mathrm{cm}$ tonsil mass without adenomegaly. Initial workup revealed slight elevation in gamma-glutamyltransferase (GGT; $67 \mathrm{U} / \mathrm{I}$ [normal: 0-40 U/I]) probably due to the patient's history of alcohol consumption. The remaining liver function tests were normal as were the renal and thyroid function tests. Serology tests for hepatitis B and C and HIV were negative.

First-line immunotherapy was started on intravenous pembrolizumab 200 mg every 3 weeks. Following her first infusion, the patient noted grade 2 dysorexia and epigastralgia. Given the weightloss (-8\%) and the anorexia, the placement of a nasogastric tube is performed. Following cycle 2, the patient reported worsening dysorexia but pain improvement. At this time, positron emission tomography-computed tomography revealed a stable disease with a slight improvement of the SCC of the tonsil and the pulmonary mass but a slight progression of the subscapular lymph node. Without a significant progression, pembrolizumab is continued, and an association with oropharynx and subscapular lymph node radiotherapy was decided.

Less than 3 weeks after the second infusion, blood tests revealed a massive increase in liver transaminases with a grade 4 toxicity (GGT $=1,647 \mathrm{U} / \mathrm{I}=40 \times \mathrm{ULN}$ and AST $=509 \mathrm{U} / \mathrm{I}=$ $14 \times$ ULN) (shown in Table 1). During a few days, liver perturbations were continuous, but the patient remained totally asymptomatic with a very good performance status of 1 . Due to this poor clinical presentation, the medical background of our subject, and the precocity of the biological variations, we wrongly thought that alcohol consumption or enteral alimentation could have been responsible.

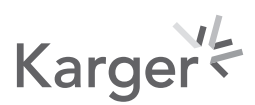


Fig. 1. PDL1 immunohistochemical staining of patient: more than $80 \%$ of the tumor is infiltrated by PDL1 positive cells. $\times 10$.

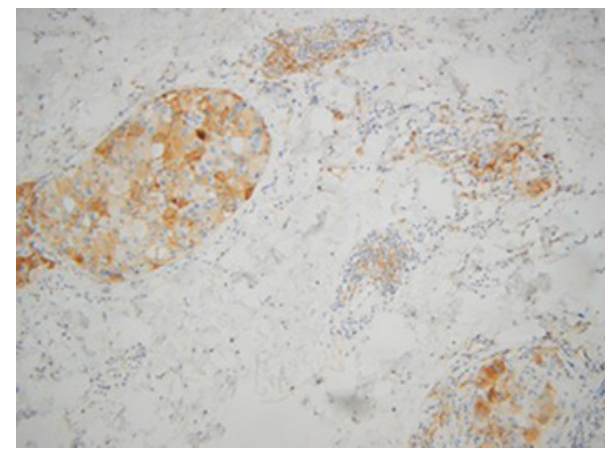

Table 1. NCI-CTCAE, version 5

\begin{tabular}{llllll}
\hline Adverse event & Grade 1 & Grade 2 & Grade 3 & Grade 4 & Grade 5 \\
\hline ALT or AST & $1-3 \times$ ULN & $3-5 \times$ ULN & $5-20 \times$ ULN & $>20 \times$ ULN & - \\
PAL or GGT & $1-2.5 \times$ ULN & $2.5-5 \times$ ULN & $5-20 \times$ ULN & $>20 \times$ ULN & - \\
BIL & $1-1.5 \times$ ULN & $1.5-3.0 \times$ ULN & $3.0-10.0 \times$ ULN & $>10 \times$ ULN & - \\
\hline
\end{tabular}

AST, aspartate aminotransferase; ALT, alanine aminotransferase; PAL, alkaline phosphatase; GGT, gamma-glutamyltransferase; BIL, bilirubin; ULN, upper limit of normal; NCI-CTCAE, National Cancer Institute's Common Terminology Criteria for Adverse Events.

Pembrolizumab treatment was delayed, and enteral nutrition was stopped. Liver transaminases return spontaneously to within subnormal limits after 8 days. Cycle 3 of pembrolizumab was performed a week later, and a gastrostomy feeding tube was placed. Oropharynx and subscapular lymph node radiotherapy were started a week after the third pembrolizumab treatment. The patient received 66 Gy in 2.2 Gy fractions (30 fractions administered using intensity-modulated radiotherapy). Three weeks after this third infusion, another blood test revealed a massive increase in liver transaminases with a grade 4 toxicity (shown in Table 2; Fig. 2), about twice as significant as the previous one (AST $=18 \times$ ULN, ALT $=15 \times$ ULN, and GGT = 19× ULN, numerical values in Table 2).

Pembrolizumab was stopped, corticosteroids were started (prednisolone $2 \mathrm{mg} / \mathrm{kg}$ PO for 2 weeks), and liver biopsy was performed. Hepatic transaminases responded quickly, but a furtive recurrence occurred after biopsies. Corticosteroid treatment was extended 2 weeks for a total treatment duration of 1 month (shown in Table 2). We chose and maintained the oral way because the patient was still asymptomatic, and the efficacy was rapidly observed. The biopsies results revealed periportal hepatitis infiltrated with eosinophils and some others inflammatory cells, modest interface, hepatitis and intrahepatocytic cholestasis but no lobular hepatitis (shown in Fig. 3). These findings were consistent with an immune-related hepatitis. Nothing was consistent with a hepatopathy linked to enteral nutrition or an alcoholic hepatitis.

Hepatic transaminases quasi-normalized 1 month from the start of corticosteroids without relapse. Corticosteroid treatment was stopped progressively. Pembrolizumab was not restarted. Closer monitoring was performed, and no further liver dysfunction occurred after stopping corticosteroids.

At this time, 18 months after stopping pembrolizumab, restaging studies revealed no evidence of SCC of the tonsil and no evidence of distant metastases. Only the pulmonary lesion persists with a partial response at the pembrolizumab treatment (shown in Fig. 4). 


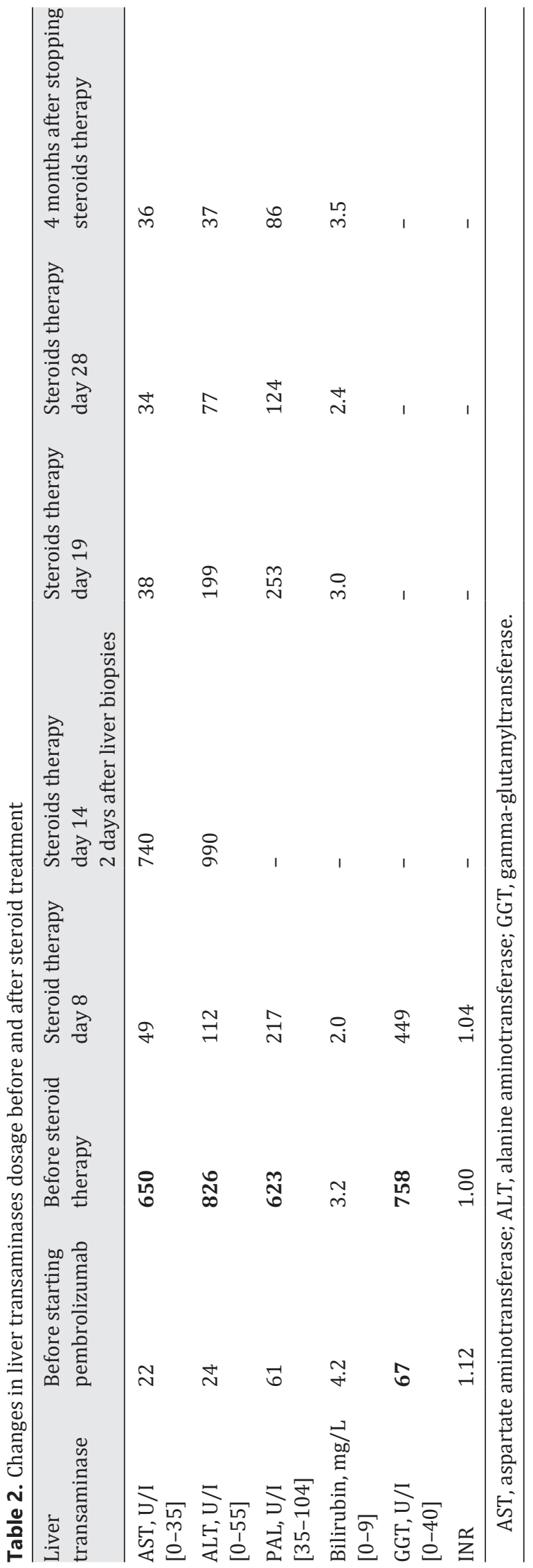




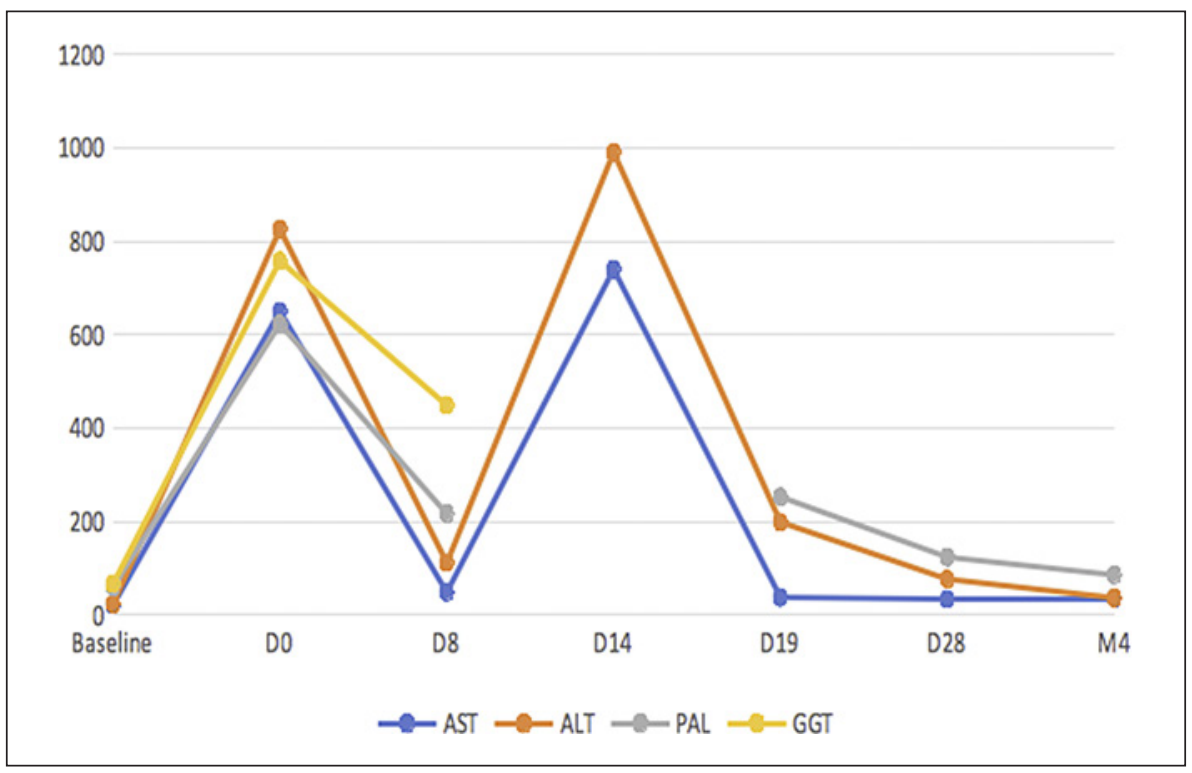

Fig. 2. Changes in liver transaminases dosage (U/I) before and after corticosteroid initiation (D0). AST, aspartate aminotransferase; ALT, alanine aminotransferase; GGT, gamma-glutamyltransferase.

Fig. 3. Immune-mediated hepatitis: patient histologic presentation with eosinophilic infiltration. Hematoxylin and eosin stain. $\times 200$.

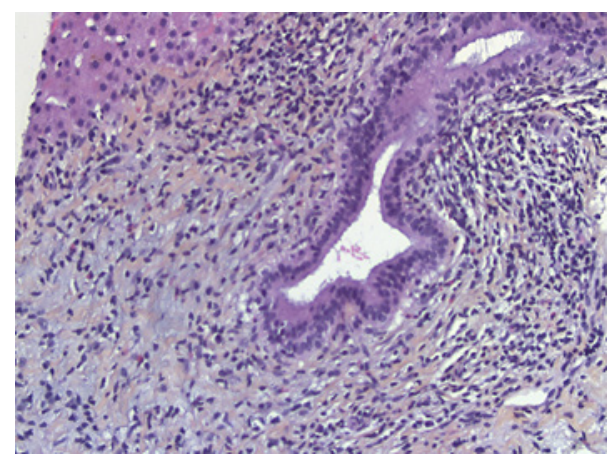

\section{Discussion}

Anti-PD1 antibodies represent an effective treatment option for metastatic NSCLC and unresectable head and neck SCC, which overexpressed PDL1. Targeting its immune checkpoint reactivates cytotoxic $\mathrm{T}$ lymphocytes, allowing the immune system to destroy cancer cells [15]. At present, immune checkpoint inhibitors have become first-line therapy for a variety of advanced malignancies. However, reactivate cytotoxic T lymphocytes may also results in immune-related adverse events (irAEs) in a proportion of patients as reactivated $\mathrm{T}$ cells attack other tissues, potentially in any organ system. Although there are a wide range of known irAEs associated with checkpoint inhibitors, cutaneous, thyroid, and gastrointestinal AEs are among the most commonly observed toxicities $[16,17]$. The mechanisms of irAEs are still incompletely understood as well the reason for predominance of these toxicities. Gastrointestinal irAEs might be due to a cross-reaction between tumor antigens and normal tissue antigens of the gastrointestinal tract and/or an overlap between microbial epitopes and tumor neoantigens $[15,18]$.

A permanent discontinuation of therapy due to side effects has been reported in $5 \%$ of patients treated with anti-PD1/PDL1 antibodies [19] and grade 3-4 IMH in only 1.1\% 

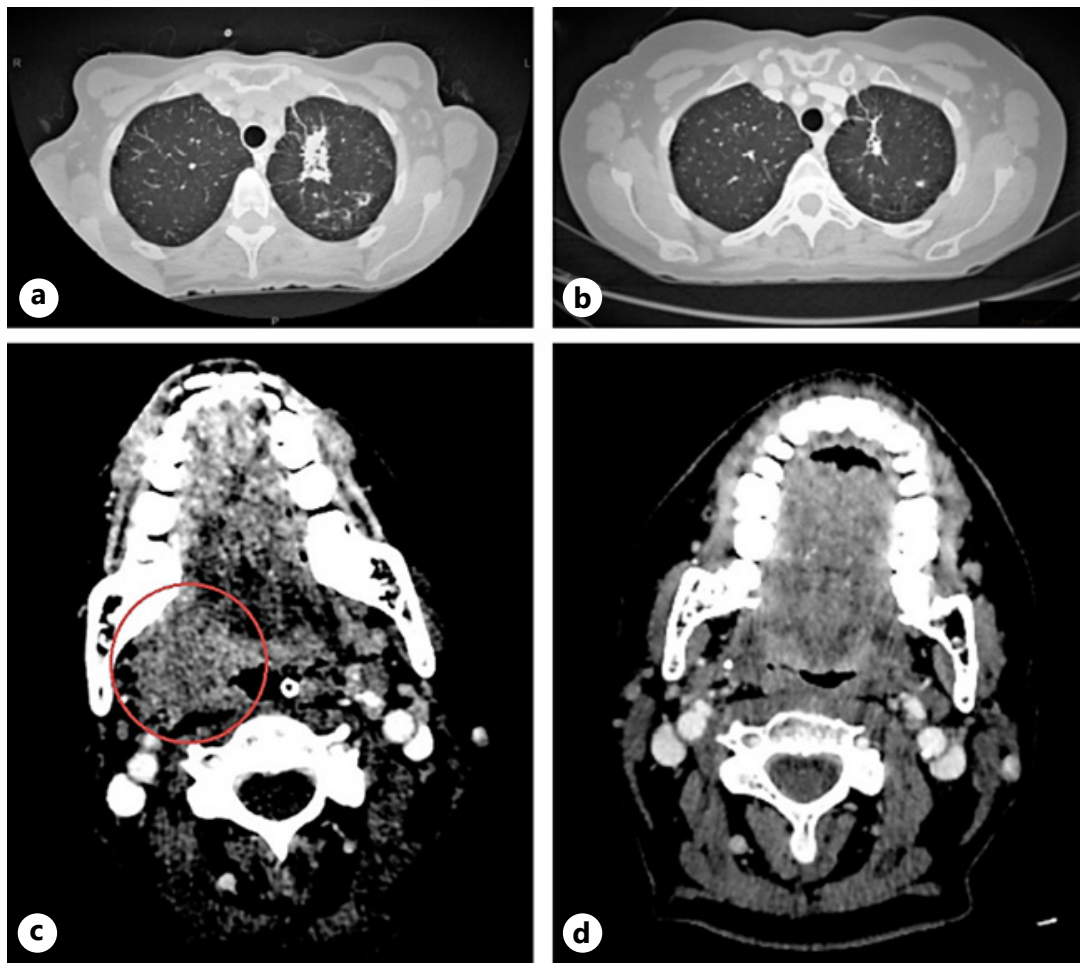

Fig. 4. CT scan response with pembrolizumab in the patient with stage 4 lung adenocarcinoma. Pulmonary lesion pretreatment (a) and 13-month posttreatment (b) showing reduction in tumor mass. Tonsil lesion pretreatment (c) and 13-month posttreatment (d) showing a complete response.

[0.5-1.7] [15]. This percentage increases significantly in patients receiving combination immunotherapy with anti-PD1/PDL1 and CTLA-4 checkpoint inhibitors, up to $20 \%$ [20]. We report an uncommon case of a metastatic NSCLC patient managed with first-line therapy with pembrolizumab who showed a very good treatment response, despite the few injections of pembrolizumab, his discontinuation and corticosteroid therapy for grade $4 \mathrm{IMH}$.

Making the diagnosis of IMH can be challenging in the context of potentially alcoholic patients under enteral nutrition. In our case, first, liver transaminase increases (after the second pembrolizumab injection) have been attributed to enteral nutrition and/or alcohol consumption in the first hypothesis. Pembrolizumab delay and enteral nutrition discontinuation allowed recovering subnormal liver transaminases values (grade 1, 2 weeks after stopping enteral nutrition). Finally, IMH was confirmed histologically after the second liver transaminases increase and managed effectively with corticosteroids. The periportal hepatitis, the liver dysfunction after immunotherapy, and the recovery with corticosteroids signed our diagnosis, and no liver autoantibodies were tested.

Although the incidence of liver dysfunction caused by anti-PD1 therapy is much lower than gastrointestinal or cutaneous AEs, it seems essential to suspect it the earlier in order to confirm and manage it as soon as possible. The lack of knowledge about this kind of toxic effect could lead to fulminant hepatitis and worse, and it is important to learn that major liver dysfunction could occur just after the first anti-PD1 perfusions. ASCO and ESMO guidelines for immunotherapy-related hepatitis recommend permanently checkpoint inhibitor discontinuation for grade 3 or 4 transaminase elevation [21,22]. Currently, the management of grade 4 liver toxicities requires high-dose intravenous glucocorticoids for 24-48 h, followed by an oral steroid taper with prednisolone $1-2 \mathrm{mg} / \mathrm{kg}$ over at least 1 month $[21,23]$. 
However, ASCO guidelines do not specify oral or IV administration [22]. Our patient receive only oral steroid. The use of steroids is based on the mechanisms of immune-related colitis, highly reminiscent of idiopathic intestinal inflammatory disease. However, corticosteroids might not be necessary for all toxicities related to immune checkpoint inhibitors. Accordingly and despite the ASCO and ESMO recommendations for corticosteroid therapy, in current practice, the management of IMH appears heterogeneous, from abstention to corticosteroids or immunosuppressive drugs [21, 24-26]. Some authors even report the absence of grade 3-4 hepatitis after reintroduction of immunotherapy [26]. In our case, the patient experienced a spontaneous improvement in liver tests without any corticosteroid therapy, during the first IMH episode. Nevertheless, after 2 IMH events, Pembrolizumab was definitively stopped. Usually, permanent discontinuation of immune checkpoint inhibitor is recommended with confirmed grade 3-4 toxicities [21,22, 27]. In the event of IMH suspicion, immunotherapy interruption and steroid initiation should not be delayed. Late management can be harmful for patients.

In our patient's case, despite IMH and steroid exposure, a very good response to anti-PD1 therapy was observed, with no evidence of progression 18 months after treatment discontinuation. These findings are consistent with recent exploratory analyses on the association between adverse events and outcomes in patients receiving PD1 or PDL1 antibodies $[28,29]$. These studies report that patients who reported irAEs were more likely to respond to anti-PD1/PDL1 treatment, without any relationship with the duration of exposure. Moreover, systemic corticosteroid use did not appear to affect the duration of response. These findings are reassuring in the context of the theoretical concern that corticosteroids may decrease the efficacy of checkpoint inhibitors by suppressing the antineoplastic immune response [30]. It should be also noted that a combination of radiotherapy and immunotherapy can improve the overall treatment response by triggering a nontargeted tumor control event called the abscopal effect [31]. The mechanism of the abscopal effect is not yet completely understood, but this synergistic antitumor effect has been investigated in many clinical studies [32]. It has even been reported once while radiotherapy was delivered after immunotherapy [33]. The abscopal effect of radiotherapy potentiated by immune checkpoint inhibitors, by promoting the release of tumor antigens and the crosspresentation of tumor-derived antigens to T cells, could explain the excellent response to our patient's treatment for lung cancer.

In summary, IMH is an uncommon but known complication of immune checkpoint inhibitor, and clinicians should suspect it early in their patients for managing it effectively. In addition, corticosteroids should not be delayed (because of the fear of reducing treatment effectiveness), and early discontinuation is not significant of a poor prognosis.

\section{Acknowledgements}

We thank all physicians who participate in data collection, and we thank the patient for accepting the publication.

\section{Statement of Ethics}

This study protocol was reviewed, and the need for approval was waived by our local committee. Written informed consent was obtained from the patient for publication of this case report and any accompanying images. A copy of the written consent is available for review by the editor-in-chief of this journal.

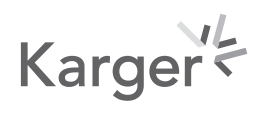




\section{Conflict of Interest Statement}

The authors have no conflict of interest to declare.

\section{Funding Sources}

This manuscript did not receive any funding.

\section{Author Contributions}

L.V. collected the data and drafted the manuscript. B.C., A.S., and F.-R.V. critically revised the manuscript for important intellectual content.

\section{Data Availability Statement}

All data generated or analysed during this study are included in this article. Further inquiries can be directed to the corresponding author.

\section{References}

1 Kwok G, Yau TC, Chiu JW, Tse E, Kwong YL. Pembrolizumab (keytruda). Hum Vaccin Immunother. 2016; 12(11):2777-89.

2 US Food and Drug. FDA approves pembrolizumab for first-line treatment of head and neck squamous cell carcinoma. [cited 2020 March 5]. Available from: https://www.fda.gov/drugs/resources-informationapproved-drugs/.

3 Wherry EJ. T cell exhaustion. Nat Immunol. 2011;12(6):492-9.

4 Nguyen LT, Ohashi PS. Clinical blockade of PD1 and LAG3: potential mechanisms of action. Nat Rev Immunol. 2015;15(1):45-56.

5 Topalian SL, Drake CG, Pardoll DM. Immune checkpoint blockade: a common denominator approach to cancer therapy. Cancer Cell. 2015;27(4):450-61.

6 Keir ME, Butte MJ, Freeman GJ, Sharpe AH. PD-1 and its ligands in tolerance and immunity. Annu Rev Immunol. 2008;26:677-704.

7 Petri CR, Patell R, Batalini F, Rangachari D, Hallowell RW. Severe pulmonary toxicity from immune checkpoint inhibitor treated successfully with intravenous immunoglobulin: case report and review of the literature. Respir Med Case Rep. 2019;27:100834.

8 Hansen E, Sahasrabudhe D, Sievert L. A case report of insulin-dependent diabetes as immune-related toxicity of pembrolizumab: presentation, management and outcome. Cancer Immunol Immunother. 2016;65(6): 765-7.

9 Smith-cohn MA, Gill D, Voorhies BN, Agarwal N, Garrido-Laguna I. Case report: pembrolizumab-induced Type 1 diabetes in a patient with metastatic cholangiocarcinoma. Immunotherapy. 2017;9(10):797-804.

10 Robinson SD, Lai C, Hotton G, Anand G. Life threatening pembrolizumabinduced myositis in a patient treated for advanced adenocarcinoma of the lung. Acute Med. 2019;18(3):197-9.

11 Sibaud V, David I, Lamant L, Resseguier S, Radut R, Attal J, et al. Acute skin reaction suggestive of pembrolizumab-induced radiosensitization. Melanoma Res. 2015;25(6):555-8.

12 Gaudy C, Clévy C, Monestier S, Dubois N, Préau Y, Mallet S, et al. Anti-PD1 pembrolizumab can induce exceptional fulminant Type 1 diabetes. Diabetes Care. 2015;38(11):e182-3.

13 Iskandar A, Hwang A, Dasanu CA. Polymyalgia rheumatica due to pembrolizumab therapy. J Oncol Pharm Pract. 2019;25(5):1282-4.

14 Bhave P, Buckle A, Sandhu S, Sood S. Mortality due to immunotherapy related hepatitis. J Hepatol. 2018;69(4): 976-8.

15 Shivaji UN, Jeffery L, Gui X, Smith SCL, Ahmad OF, Akbar A, et al. Immune checkpoint inhibitor-associated gastrointestinal and hepatic adverse events and their management. Therap Adv Gastroenterol. 2019 Nov 5; 12:1756284819884196.

16 Su Q, Zhang X, Shen X, Hou Y, Sun Z, Gao ZH. Risk of immune related colitis with PD-1/PD-L1 inhibitors versus chemotherapy in solid tumors: systems assessment. J Cancer. 2018;9(9):1614-22. 
17 Sibaud V. Dermatologic reactions to immune checkpoint inhibitors: skin toxicities and immunotherapy. Am J Clin Dermatol. 2018;19(3):345-61.

18 Khan Z, Hammer C, Guardino E, Chandler GS, Albert ML. Mechanisms of immune-related adverse events associated with immune checkpoint blockade: using germline genetics to develop a personalized approach. Genome Med. 2019 Jun 20;11(1):39.

19 Topalian SL, Hodi FS, Brahmer JR, Gettinger SN, Smith DC, McDermott DF, et al. Safety, activity, and immune correlates of anti-PD-1 antibody in cancer. N Engl J Med. 2012;366(26):2443-54.

20 Larkin J, Hodi FS, Wolchok JD. Combined nivolumab and ipilimumab or monotherapy in untreated melanoma. N Engl J Med. 2015;373(13):1270-1.

21 Haanen JBAG, Carbonnel F, Robert C, Kerr KM, Peters S, Larkin J, et al. Management of toxicities from immunotherapy: ESMO Clinical Practice Guidelines for diagnosis, treatment and follow-up. Ann Oncol. 2017; 28(Suppl 4):iv119-42.

22 NCCN clinical practice guidelines in Oncology (NCCN Guidelines $®$ ) in partnership with the American society of clinical Oncology (ASCO) [Internet]. Management of Immunotherapy-Related Toxicities [Cited 2020 March 5]. Available from: http://www.omedit-idf.fr/wp-content/uploads/2018/05/NCCN-2018-Reco-EI-immunotherapies.pdf.

23 Eigentler TK, Hassel JC, Berking C, Aberle J, Bachmann O, Grünwald V, et al. Diagnosis, monitoring and management of immune-related adverse drug reactions of anti-PD-1 antibody therapy. Cancer Treat Rev. 2016;45:7-18.

24 Puzanov I, Diab A, Abdallah K, Bingham CO 3rd, Brogdon C, Dadu R, et al. Managing toxicities associated with immune checkpoint inhibitors: consensus recommendations from the Society for Immunotherapy of Cancer (SITC) Toxicity Management Working Group. J Immunother Cancer. 2017;5(1):95.

25 Gauci ML, Baroudjian B, Zeboulon C, Pages C, Poté N, Roux O, et al. Immune-related hepatitis with immunotherapy: are corticosteroids always needed? J Hepatol. 2018;69(2):548-50.

26 De martin E, Michot JM, Papouin B, Champiat S, Mateus C, Lambotte 0, et al. Characterization of liver injury induced by cancer immunotherapy using immune checkpoint inhibitors. J Hepatol. 2018;68(6):1181-90.

27 Brahmer JR, Lacchetti C, Thompson JA. Management of immune-related adverse events in patients treated with immune checkpoint inhibitor therapy: American society of clinical oncology clinical practice guideline summary. J Oncol Pract. 2018;14(4):247-9.

28 Maher VE, Fernandes LL, Weinstock C, Tang S, Agarwal S, Brave M, et al. Analysis of the association between adverse events and outcome in patients receiving a programmed death protein 1 or programmed death ligand 1 antibody. J Clin Oncol. 2019;37(30):2730-7.

29 Shafgat H, Gourdin T, Sion A. Immune-related adverse events are linked with improved progression-free survival in patients receiving anti-PD-1/PD-L1 therapy. Semin Oncol. 2018;45(3):156-63.

30 Oppong E, Cato AC. Effects of glucocorticoids in the immune system. In: Wang J-C, Harris C, editors. Glucocorticoid signaling. New York, NY, USA: Springer; 2015. Vol. 872; p. 217-33. Advances in Experimental Medicine and Biology.

31 Akbor M, Hung KF, Yang YP, Chou SJ, Tsai PH, Chien CS, et al. Immunotherapy orchestrates radiotherapy in composing abscopal effects: a strategic review in metastatic head and neck cancer. J Chin Med Assoc. 2020; 83(2):113-6.

32 Liu Y, Dong Y, Kong L, Shi F, Zhu H, Yu J. Abscopal effect of radiotherapy combined with immune checkpoint inhibitors. J Hematol Oncol. 2018;11(1):104.

33 Grimaldi AM, Simeone E, Giannarelli D, Muto P, Falivene S, Borzillo V, et al. Abscopal effects of radiotherapy on advanced melanoma patients who progressed after ipilimumab immunotherapy. Oncoimmunology. 2014; 3:e28780. 